\title{
Molecular and cellular pathogenesis of autosomal recessive polycystic kidney disease
}

L.F. Menezes ${ }^{1,2}$ and L.F. Onuchic ${ }^{1}$
Correspondence

L.F. Onuchic

Departamento de Clínica Médica

Faculdade de Medicina

Universidade de São Paulo

Av. Dr. Arnaldo, 455, Sala 3310

01246-903 São Paulo, SP

Brasil

E-mail: lonuchic@lim12.fm.usp.br

Research supported by FAPESP (Nos. 2000/00280-3 and 2004/02622-0 to L.F. Onuchic). $\ldots \ldots \ldots \ldots \ldots \ldots \ldots \ldots$

Received May 4, 2006 Accepted August 29, 2006
1Disciplina de Nefrologia, Departamento de Clínica Médica, Faculdade de Medicina, Universidade de São Paulo, São Paulo, SP, Brasil ${ }^{2}$ Department of Medicine, Division of Nephrology, Johns Hopkins University School of Medicine, Baltimore, MD, USA
Abstract

Autosomal recessive polycystic kidney disease (ARPKD) is an inherited disease characterized by a malformation complex which includes cystically dilated tubules in the kidneys and ductal plate malformation in the liver. The disorder is observed primarily in infancy and childhood, being responsible for significant pediatric morbidity and mortality. All typical forms of ARPKD are caused by mutations in a single gene, PKHDl (polycystic kidney and hepatic disease 1). This gene has a minimum of 86 exons, assembled into multiple differentially spliced transcripts and has its highest level of expression in kidney, pancreas and liver. Mutational analyses revealed that all patients with both mutations associated with truncation of the longest open reading frame-encoded protein displayed the severe phenotype. This product, polyductin, is a 4,074-amino acid protein expressed in the cytoplasm, plasma membrane and primary apical cilia, a structure that has been implicated in the pathogenesis of different polycystic kidney diseases. In fact, cholangiocytes isolated from an ARPKD rat model develop shorter and dysmorphic cilia, suggesting polyductin to be important for normal ciliary morphology. Polyductin seems also to participate in tubule morphogenesis and cell mitotic orientation along the tubular axis. The recent advances in the understanding of in vitro and animal models of polycystic kidney diseases have shed light on the molecular and cellular mechanisms of cyst formation and progression, allowing the initiation of therapeutic strategy designing and promising perspectives for ARPKD patients. It is notable that vasopressin V2 receptor antagonists can inhibit/halt the renal cystic disease progression in an orthologous rat model of human ARPKD.

\section{Introduction}

Autosomal recessive polycystic kidney disease (ARPKD) is a hereditary disorder that affects the kidneys and the biliary tract. This disease is a major cause of pediatric renal cystic disease, often presenting as a devastating condition. Its clinical spectrum,
Key words

- Autosomal recessive polycystic kidney disease

- ARPKD

- PKHD1 gene

- Polyductin/Fibrocystin

- Polycystic kidney disease

- Primary cilium

- Cystogenesis however, is highly variable, ranging from a severe perinatal phenotype to later onset and milder forms.

\section{Epidemiology and natural history}

ARPKD has an estimated incidence of 1 in 20,000 live births (1). The most severely 
affected cases are identified in utero and characterized by the development of enlarged and echogenic kidneys in diseased fetuses. The decreased fetal urine output, in turn, leads to oligohydramnios and, possibly, to pulmonary hypoplasia.

The reported incidence of severe pulmonary problems in ARPKD neonates varies from 13 to $75 \%$ depending on the nature of the analyzed patient population (2). Recent observations have suggested that approximately $30 \%$ of the affected neonates die shortly after birth as a result of respiratory insufficiency (3). In a study including 166 North American patients, Guay-Woodford and Desmond (1) reported an overall survival of $85.8 \%$ at 1 month, $78.6 \%$ at 1 year, and $74.6 \%$ at 5 years, with the highest mortality rate $(58 \%)$ occurring within the first
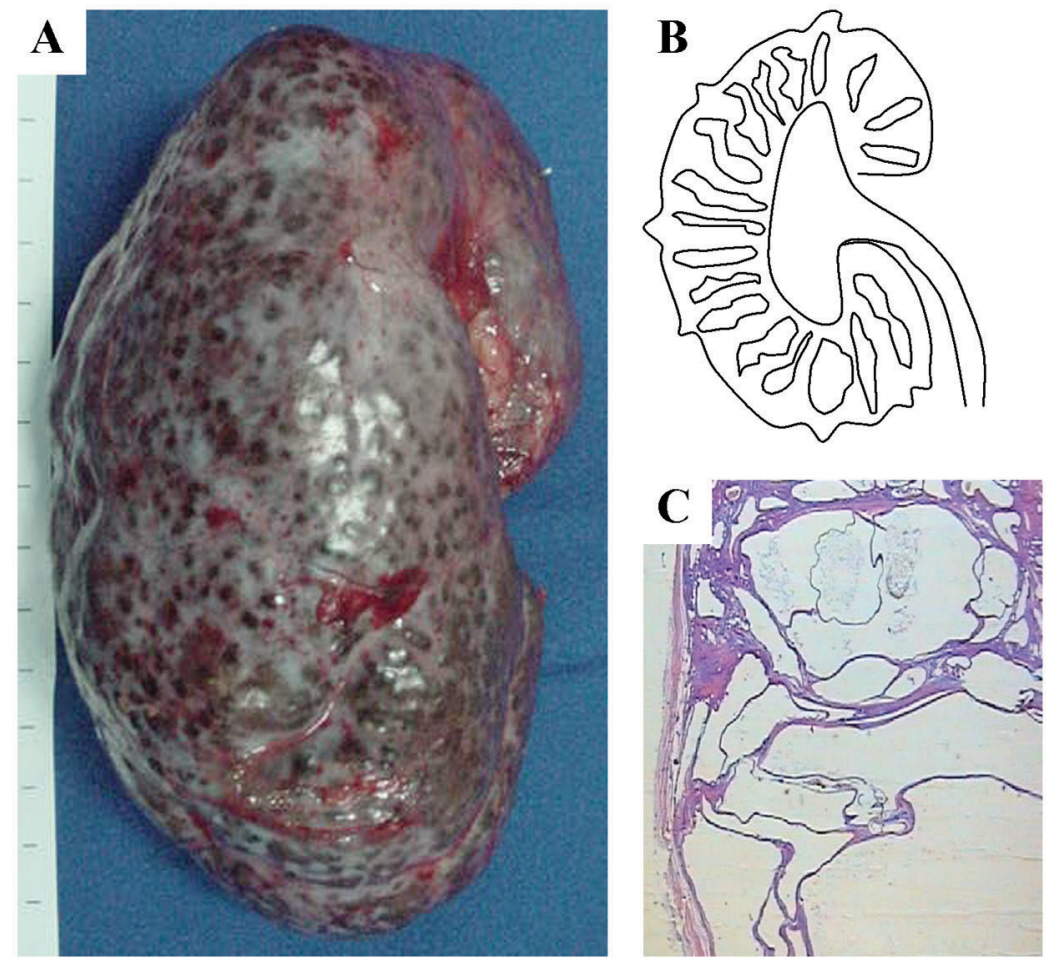

Figure 1. Renal pathology of autosomal recessive polycystic kidney disease. $A$, Kidney of a 2-year-old ARPKD-affected child. Macroscopically, the organ displays multiple small cysts on its surface and is severely enlarged, measuring $15 \mathrm{~cm}$ in its longitudinal axis (Gift of Dr. Francisco Tibor Dénes). B, Drawing representing the diffuse and radial distribution of dilated collecting ducts throughout the cortex and medulla. $C$, ARPKD renal histology, showing dilation of collecting ducts. month of life. The authors acknowledge, however, that this study probably underestimated the early mortality rate.

In the subset of patients who survive the perinatal period, morbidity and mortality are mainly associated with systemic hypertension, progressive renal insufficiency, and portal hypertension secondary to portal-tract fibrosis. ARPKD, in fact, is invariably associated with biliary dysgenesis. A more recent study, following 164 neonatal survivors, reported 1- and 10-year survival rates of 85 and $82 \%$, respectively, in this patient population (4). In this group, chronic renal failure was first detected at a mean age of 4 years, while the actuarial renal survival rates were $86 \%$ at 5 years, $71 \%$ at 10 years and $42 \%$ at 20 years.

\section{Pathology}

The ARPKD renal histopathology is characterized by bilateral and symmetric involvement. The kidney can be massively enlarged, reaching up to 10-fold its original size, with multiple 1-2-mm cysts on its surface (5). In severely affected kidneys, radially distributed dilated and fusiform tubules fill the cortex and medulla (Figure 1). The cystic epithelium is composed of a uniform, singlecell, and cuboidal layer. Cystic tubules are virtually always derived from collecting ducts and communicate freely with their corresponding non-cystic tubule segments (5).

The hepatic histopathology is characterized by ductal plate malformation (6). The portal tract often retains its original embryonic architecture, with small, distorted bile ducts located at the periphery of the portal space. Portal ducts eventually become tortuous and cystic, encircled by variable degrees of fibrosis. When larger biliary ducts are also dilated, the disorder is called Caroli's disease. In some cases, cholangitis leads to a gradual replacement of the immature ducts by fibrosis, resulting in a condition known as hepatic fibrosis. The association of hepatic 
fibrosis with Caroli's disease is called Caroli's syndrome.

\section{Genetics}

All typical forms of ARPKD are caused by mutations in a single gene, $P K H D 1$ (polycystic kidney and hepatic disease 1). Genetic linkage analyses allowed the ARPKD locus to be initially localized to the chromosome region 6p21.1-p12 $(7,8)$. The later generation of a series of physical and genetic maps, followed by recombination mapping, led to the refinement of the critical interval to less than $1 \mathrm{Mb}$ (9). This strategy allowed the construction of a candidate region transcriptional map, using database searches, cDNA library screening, bioinformatic tools, RT-PCR, and Northern blot analyses (10). Expressed sequences were screened for mu- tations in ARPKD patient samples using DHPLC and DNA sequencing. Finally, the identification of disease-associated genetic variants in different portions of a large transcript led to the identification and characterization of the ARPKD gene, PKHDI (10). Simultaneously, another group independently identified $P K H D I$ by characterizing its orthologue in the polycystic kidney ( $p c k$ ) rat model (11).

\section{The PKHD1 gene}

PKHDl is a very large and complex gene. It spans a genomic segment of over $469 \mathrm{~kb}$ and has a minimum of 86 exons, assembled into multiple differentially spliced transcripts (Figure 2) (10). A 67-exon transcript, 12.6$\mathrm{kb}$ long, encodes the gene's putative longest open reading frame (ORF). Northern blot
A

Exons in the longest pontential ORF

B

Alternative exons

C

Amplified products associated with alternative transcripts

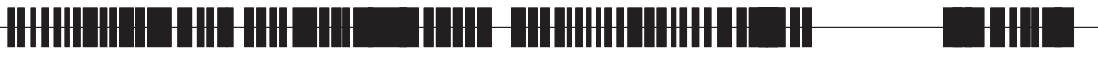

Non-overlapping alternative exons

Overlapping alternative exons
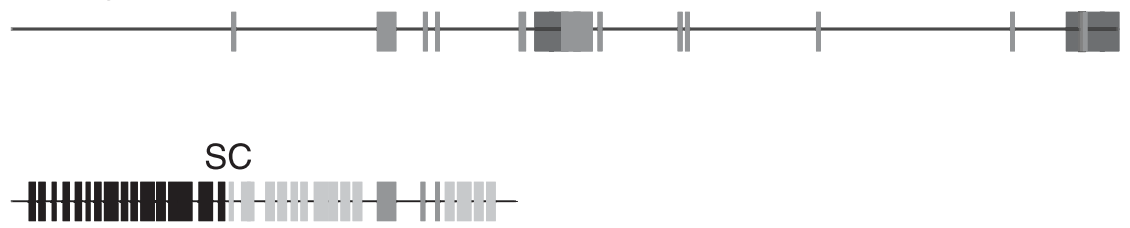

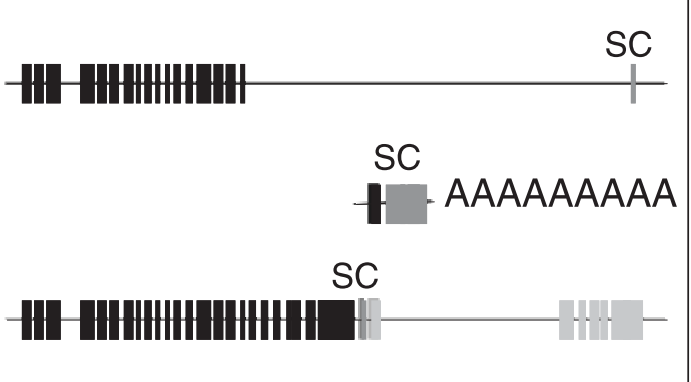

Figure 2. The PKHD1 gene structure. The gene has 86 exons so far identified; 71 are non-overlapping while 15 are exons with alternative splicing boundaries ( $\mathrm{A}$ and $\mathrm{B}$ ). The putative longest open reading frame (ORF) transcript comprises 67 exons (A). Preliminary analyses suggest that $P K H D 1$ encodes a high number of alternative transcripts. Four examples of differential assembling are shown at the bottom of the figure (C). SC: approximate location of the stop codon; dark gray: alternative exons; light gray: noncoding exons in the corresponding transcripts. 
analysis in human tissues revealed a broad and smeared signal, consistent with the existence of multiple different-size transcripts. PKHDl has its highest level of expression in human fetal and adult kidneys, but lower levels were also detected in pancreas and even lower levels in human fetal and adult liver (10). If a significant number of the alternatively spliced products are translated, their exon arrangements predict that both membrane-bound and soluble proteins should be produced (10). In addition, it should be noted that a PKHDI homologue, the $P K H D L 1$ gene, was recently identified and shown to share the complex splicing property with $P K H D 1$. While its putative longest ORF is equally expected to yield a membrane-bound protein, the gene is predicted to encode membrane and soluble products, including the secreted protein D86 (12). These findings strongly suggest biological relevance for the PKHDI-encoded soluble products. The identified protein product encoded by $P K H D 1$ has been called polyductin (10) or fibrocystin (11).

The PKHDl mouse orthologue, Pkhdl, was shown to conserve the basic features of its human counterpart. It is a very large gene, extending over a 500-kb genomic region, with its putative longest ORF also encoded by a 67 -exon transcript (13). It also presents a complex splicing pattern, giving rise to multiple transcripts. Northern blot analysis showed that $P k h d l$ is expressed in kidney and weakly in liver, heart, stomach, intestine, muscle, uterus, and placenta. Interestingly, studies using two different cDNA probes, derived from exons 5 and 41, revealed a small, $\sim 1-\mathrm{kb}$ transcript in the testis, detected exclusively with the exon 41-related probe (13).

In situ hybridization analyses in developing and adult mouse tissues showed high levels of expression in renal tubular structures in the mesonephros (day E12.5), branching ureteric bud and collecting ducts. Other structures in which Pkhdl expression was observed included developing biliary ducts, muscular wall of large vessels, primordial testis, dorsal root ganglia, embryonic lung mesenchyme, pancreatic ducts, developing trachea, and skeletal muscle. Similarly to the Northern blot results, in situ hybridization studies revealed different splicing profiles in distinct tissue structures. Transcripts expressed in the wall of large vessels, developing lung or trachea include exon 41 but lack exon 5, while those present in kidney and liver comprise both exons (13). Interestingly, a genetically modified mouse lacking exon 40 , which is part of the longest $P k h d l$ transcript expressed in kidney and liver, developed cystic biliary dysgenesis but no morphologically abnormal kidney phenotype (14).

\section{PKHD1 mutation analyses in autosomal recessive polycystic kidney disease}

Several studies have recently analyzed mutations in the PKHDI longest ORF transcript in ARPKD patients, providing initial genotype-phenotype correlations $(10,11,15$ 17). All patients with both mutations associated with truncation of the longest ORFencoded protein expressed the severe phenotype. In addition, $P K H D I$ mutations have also been reported in patients with congenital hepatic fibrosis and Caroli's disease, with minimal or no kidney involvement $(11,17)$.

All studies found that the mutations are scattered throughout the gene. The overall mutation detection rate in ARPKD patients significantly varied between the severe and the moderate phenotypic groups, and among the studies. The highest detection rate was obtained in a more recent study, reaching $82.7 \%$ (3). These investigators evaluated all 86 predicted exons, including the 67 that are part of the longest ORF transcript and the 19 alternative exons. According to these researchers, the prediction of whether a sequence variant is pathogenic should rely on the following criteria: 1) a potential chain-terminating effect 
on the longest predicted polypeptide, 2) disruption of a canonical splice site or creation of a novel site, 3) substitution of an evolutionarily conserved amino acid in the context of the Miller and Kumar matrix, and 4) detection in $<0.5 \%$ of normal chromosomes. Missense changes that meet criterion 2 or 3 but occur in $>0.5 \%$ of normal chromosomes should be defined as unclassified. Interestingly, Sharp and colleagues (3) added a new piece of information to the genotype-phenotype correlation: splice site mutations in both alleles or in combination with a truncating mutation have also always resulted in the severe perinatal phenotype.

\section{Polyductin, the PKHD1 gene product}

All but the first exon of the gene's longest ORF transcript are coding. The putative ORF, in turn, is predicted to encode a 4,074amino acid integral membrane protein (10, 11). This protein includes a putative 3,858 amino acid extracellular amino terminus, a single transmembrane (TM) domain and a short carboxyl intracellular tail (Figure 3). Polyductin is predicted to be a highly Nglycosylated protein. Structural analysis revealed that it comprises a series of immunoglobulin-like-plexin-transcription factor (IPT) domains in its amino terminus and multiple parallel $B$-helix $1(\mathrm{PbH} 1)$ repeats between the last IPT and the TM domains (10). Multiple tandemly arranged IPT domains are also found in single-pass cell surface receptors that belong to the Sema superfamily of proteins, including the hepatocyte growth factor receptor and the plexins, suggesting a similar function for polyductin. The $\mathrm{PbH} 1$ repeats, on the other hand, are most commonly found in polysaccharidases and may be important for carbohydrate moiety recognition and/or modification. Polyductin's short carboxyl tail includes putative cAMP/cGMP-dependent protein kinase phosphorylation sites that may also be important for its function. It is notable that mouse polyductin presents the same general domain structure as the human product. In fact, these proteins share $73 \%$ identity over their complete length, though there are segments with considerably higher $(87 \%)$ and lower (40\%) identity values (13).

Polyductin was shown to be an over 440$\mathrm{kDa}$ membrane-bound protein expressed in kidney, liver and pancreas (18-20). Two alternative products of $\sim 230$ and $\sim 140 \mathrm{kDa}$ have also been detected (18). Interestingly, the $140-\mathrm{kDa}$ band was shown to be expressed predominantly in soluble cellular fractions, suggesting that it may represent a secreted product. This study supports the concept that PKHD1 encodes membrane and soluble proteins. Immunohistochemistry analyses revealed staining of cortical and medullary col-

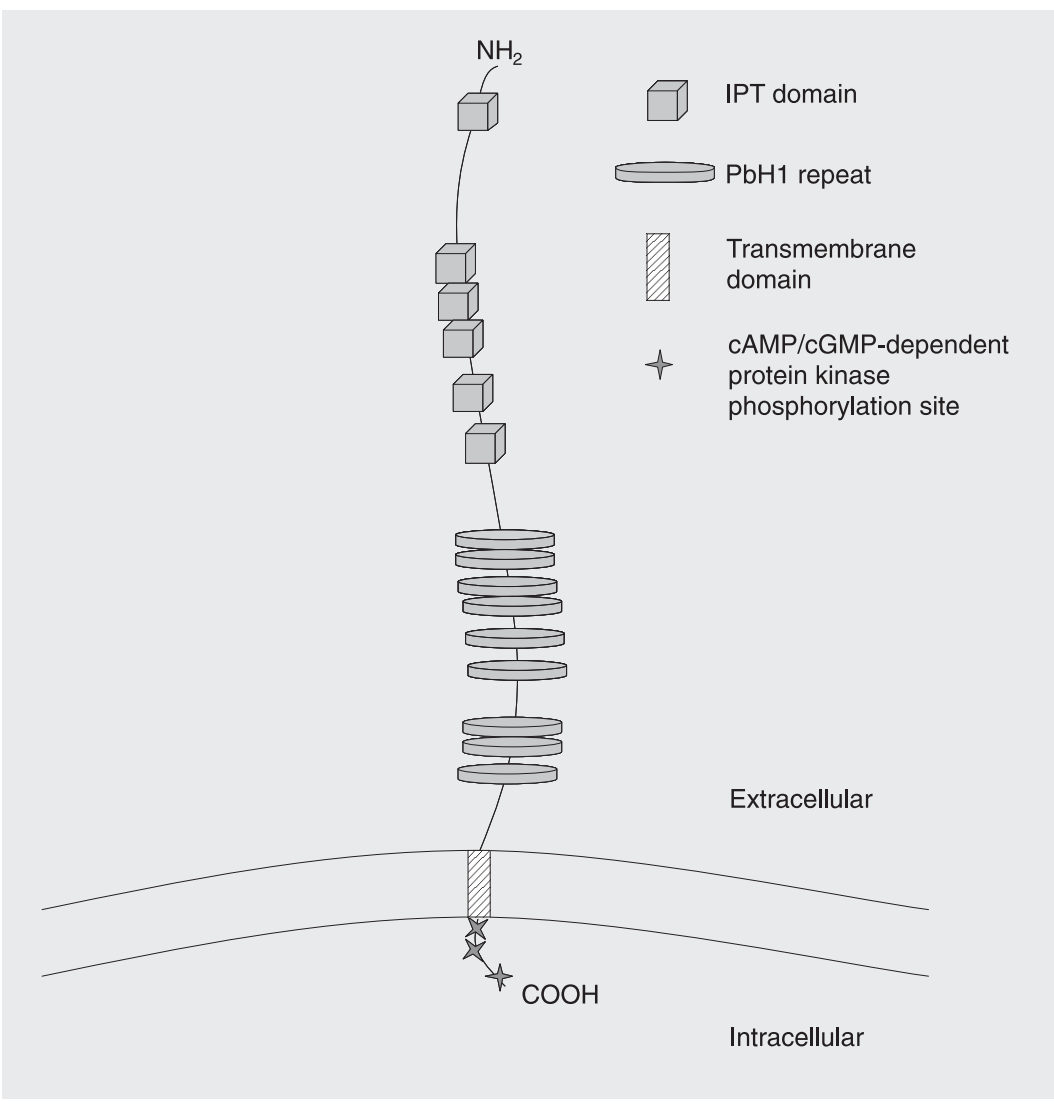

Figure 3. Domain structure of polyductin. The protein domains are identified in the figure. Only the longest open reading frame-encoded product, a membrane-associated protein, is shown in the schema. IPT = immunoglobulin-like-plexin-transcription factor; $\mathrm{PbH} 1=$ parallel ß-helix 1. 
lecting ducts and thick ascending limbs of Henle in kidney and biliary and pancreatic duct epithelia (18). In human fetus and mouse developing tissues, staining was observed in the branching ureteric bud but not in the metanephric mesenchyme, S-shaped bodies or glomeruli $(18,19)$. Liver analysis revealed a positive signal in intra- and extra-hepatic biliary ducts, pancreatic ducts and salivary gland ducts from mouse embryos. Notably, a non-overlapping staining pattern in pancreas and salivary gland acini with antibodies against different portions of polyductin was consistent with the existence of structure-specific translated products (18).

Several studies have shown that at the subcellular level polyductin is expressed in the primary apical cilia in kidney cells and cholangiocytes (18-22). This pattern has remarkable implications for ARPKD pathogenesis, as we will discuss in the next section. Polyductin is also localized at the apical membrane in collecting duct cells and in the cytoplasm of inner medullary collecting duct (IMCD) cells grown in culture. These observations suggest that, in addition to its participation in ciliary function, the protein has functional roles in other subcellular domains (18).

\section{Pathogenesis of autosomal recessive polycystic kidney disease}

\section{Role of primary apical cilia in cystogenesis}

The primary cilium is a solitary cellular structure present in almost all vertebrate cells, with the exception of bone marrow-derived cells and kidney collecting duct intercalated cells. It is a non-motile cilium, composed of nine peripherally located microtubule pairs, but lacking the central microtubule pair seen in motile cilia (23). The primary cilium develops from a modified centriole, the basal body. Its assembly and maintenance depend on the transport of proteins between the cilia and the cytoplasm, a process called intraflagellar transport (IFT). It is believed that this organelle may function as a mechanosensor, transducing flux-induced signals through intracellular calcium transients (24).

Several studies have shown that proteins translated from genes mutated in different human polycystic kidney diseases (PKD) and PKD animal models are expressed in the primary cilium. Polycystin-1 and polycystin2 , the products of $P K D 1$ (polycystic kidney disease 1) and $P K D 2$, the genes mutated in autosomal dominant polycystic kidney disease (ADPKD), have been detected in the ciliary axoneme (25). While polycystin-1 is thought to behave as a mechanosensor (26), polycystin- 2 functions as a calcium-permeable, non-selective cation channel (27). They are membrane proteins known to interact with each other by their carboxyl tails, regulating calcium cell influx. A recent study by Low and colleagues (28) shed light upon a potential transduction signaling pathway associated with ciliary mechanosensation. According to this study, polycystin-1 undergoes proteolytic cleavage, resulting in nuclear translocation of its carboxyl terminus. The interaction of the released tail with the signal transducer and activator of transcription 6 (STAT6) and the co-activator P100, in turn, stimulates STAT6-dependent gene expression, linking mechanical signaling to transcriptional changes.

The congenital polycystic kidney ( $c p k$ ) mouse has a renal phenotype that is fully expressed in homozygotes and is similar to human ARPKD (29). Cystin, the product of Cys l, the cpk gene, has also been detected at the ciliary axoneme, where it could play a role in stabilizing microtubule assembly (29). Interestingly, mice homozygous for the $c p k$ mutation treated with the microtubule stabilizer paclitaxel have a delayed progression of PKD (30).

Another recessive PKD mouse model is the Oak Ridge polycystic kidney (orpk) animal (31). The orpk allele seems to be a hypomorphic allele of $T g 737$. Polaris, the $T g 737$ gene product, is expressed in the ciliary ax- 
oneme and basal bodies, a pattern of distribution similar to that reported for proteins involved in IFT (32). It has been suggested that polaris may serve as the scaffold upon which IFT proteins assemble (32). It is interesting to note that paclitaxel treatment was ineffective in Tg737orpk homozygotes, suggesting that polaris and cystin play different roles in ciliary formation and function $(29,33)$. Despite the supporting evidence linking cilia and PKD, it should be noted that Brown and Murcia (34) showed that, while orpk mice have shortened cilia and cystic kidneys, re-expression of polaris normalizes cilium length but does not change the cystic phenotype. A recent study by Siroky and colleagues (35) provided important information to connect polaris to calcium signaling. Working with orpk collecting duct principal cells lacking primary cilia, these investigators showed unregulated cell calcium entry and increased subapical calcium concentration. Their findings suggest, moreover, that such observation can be explained on the basis of increased abundance of apical polycystin-2.

The products of genes mutated in other polycystic kidney diseases are also expressed in the ciliary axoneme and/or basal body. Such disorders include Bardet-Biedl syndrome (36) and nephronophthisis (37). Among these proteins, inversin, the product of the gene mutated in nephronophthisis type 2 (NPHP2), is particularly interesting. Recent results by Simons et al. (38) have suggested that cyst formation in the absence of inversin is caused by unopposed canonical Wnt signaling. These investigators speculate that the onset of glomerular filtration and production of primary urine lead to flow-induced up-regulation of inversin. This up-regulation terminates canonical Wnt signaling and facilitates B-cateninindependent Wnt pathways, allowing terminal differentiation of tubular epithelial cells (38). In addition, Morgan and colleagues (39) demonstrated that inversin not only localizes to cilia, but also presents a cellular distribution that changes through the cell cycle. While it is expressed in the centrosomes in prophase and in the spindle poles in metaphase and anaphase, its expression is detected in the midbody in late telophase. This observation provides a link between ciliary function and the cell cycle.

The role played by cilia in cystogenesis is also supported by Lin and co-workers (40). These investigators created a kidney-specific Kif3A knockout mouse. KIF3A is part of the heterotrimeric kinesin-II, a protein that mediates the IFT anterograde flow. In this animal model, kidney cysts developed from day P5 onward and epithelial cells lacking cilia were observed lining cystic tubules (40). In fact, these investigators reported an increased expression of $\beta$-catenin in the cytosol and nuclei of cystic kidney cells.

\section{Polyductin's relation to the primary apical cilium and its biological properties}

The ciliary expression of polyductin has been extensively studied. While Ward and colleagues (19) demonstrated that polyductin is localized to the ciliary axoneme in MadinDarby canine kidney (MDCK) cells, other groups reported expression predominantly in the basal body area in human adult and fetal kidneys, and in MDCK, IMCD3 and human embryonic kidney (HEK293) cell lines $(20,21)$. Using immunofluorescence analysis of cultured IMCD cells and immunoelectron microscopy of renal collecting duct cells, Menezes et al. (18) showed polyductin expression in both the axoneme and the basal body-axoneme transition. It has been suggested that this localization pattern might support a role for polyductin in IFT, either carried to the tip as cargo or as part of the IFT protein complex (20). Furthermore, this localization pattern seems to be independent of polycystin-1, since the polyductin expression profile is not altered in $P k d l$ null/null cells (20). A recent study has suggested that calcium-modulating cyclophilin ligand (CAML) is a polyductin binding partner by interacting with its carboxyl tail (41). Interestingly, both proteins 
are shown to co-localize in the primary cilia, basal body and apical membrane of distal nephron cells. Since CAML is a calcium signaling-related protein, these results suggest that polyductin may also contribute to the modulation of intracellular calcium.

Polyductin is apparently important for normal ciliary morphology. Analyzing microdissected cholangiocytes isolated from rat livers, Masyuk and co-workers (22) demonstrated that cilia are shorter and dysmorphic in $p c k$ rats. In addition, these investigators showed that cholangiocytes treated with Pkhdl siRNA had smaller cilia that lacked polyductin expression. Further characterization of the $p c k$ liver phenotype confirmed these findings, demonstrating that cilia are malformed, with bulbous extensions of the ciliary tip or ciliary axonemal membrane, heterogeneous in length and on average significantly shorter than normal (42).

Recent work has shown that tubule morphogenesis is associated with cell mitotic orientation along the tubular axis, revealing planar cell polarity (43). In parallel, these investigators demonstrated distortion in mitotic orientation in PKD animal models. $P k h d l$, in turn, has been shown to play a significant role in tubule morphogenesis. Inhibition of its effects by shRNA disrupted tubular morphogenesis induced in IMCD cell cultures, leading to abnormalities in cell proliferation, apoptosis, cell-cell contact, and cytoskeleton (44). Based on these analyses, such biological defects may be dependent on alterations of extracellular signal-regulated kinase (ERK) and focal adhesion kinase (FAK)-mediated signaling.

Pkhd1 transcriptional defects and cystogenesis

A recent set of studies has begun to elucidate the transcription network involving Pkhdl (45-47). Analyzing the Pkhdl gene promoter region, Hiesberger and colleagues (46) identified an evolutionarily conserved hepatocyte nuclear factor-1 (HNF-1) bind- ing site. HNF- $1 \alpha$ and HNF-1ß are transcription factors known to control the expression of several genes, especially in liver, kidney and pancreas. Mutations in $H N F-1 \alpha$ and $H N F-1 \beta$ are found in patients with maturityonset diabetes of the young types 3 and 5, respectively. Moreover, $H N F-1 \beta$ mutations have been associated with hypoplastic glomerulocystic kidney disease, cystic renal dysplasia and oligomeganephronia. Notably, Gresh and co-workers (45) demonstrated that transgenic mice with kidney-specific inactivation of $H N F-1 \beta$ developed renal cysts and bilateral ureteral dilation. Furthermore, these investigators showed that the expression of three genes involved in cystic diseases (Umod, Pkhdl and Pkd2) was decreased in mutant mice and that these genes were directly controlled by HNF-1ß (45).

Supporting evidence for a role of HNF-1ß as a $P k h d l$ transcription factor has been provided by Hiesberger and colleagues (46). These investigators cloned the $P k h d l$ promoter region and demonstrated that HNF-1ß and HNF$1 \alpha$ directly activate the $P k h d l$ promoter. They showed, in addition, that expression of a dominant negative HNF-1ß mutant in mice resulted in renal cysts (46). To further characterize the molecular mechanism involved in $P$ khd 1 regulation by HNF-1ß, this group analyzed the effects of different HNF-1ß mutants on Pkhdl promoter activation and concluded that the HNF-13 C-terminal domain is required for its activation (47). These studies, in fact, suggest a model of cystogenesis in HNF-1ß mutant disorders/models that is based on reduced Pkhdl/PKHDl expression secondary to abnormal transcription.

Epidermal growth factor receptor in autosomal recessive polycystic kidney disease

It has been suggested that abnormal expression of epidermal growth factor receptor (EGFR) in the apical surface of cystic epithelia may contribute to cyst formation and enlargement. In vitro studies analyzing cystic 
collecting duct cells derived from ARPKD patients and different recessive PKD mouse models (bpk, $c p k$, and orpk) have confirmed the abnormal apical expression of EGFR (48). Moreover, they have revealed that these receptors bind EGF, eliciting a mitogenic response. Richards and co-workers (49) elegantly demonstrated the importance of EGFR signaling in cystogenesis. These investigators engineered mice carrying both the orpk mutation and a point mutation that leads to decreased EGFR tyrosine kinase activity; these animals were reported to have a significant decrease in cyst formation. A number of studies, in fact, have confirmed the benefits of using EGFR tyrosine kinase inhibitors to attenuate cystic kidney disease in PKD animal models (50). It should be noted, however, that in the orthologous rat model of human ARPKD, the $p c k$ rat, EGFR tyrosine kinase inhibitors were not protective (51).

In addition to its role in the kidney phenotype of recessive PKD models, EGFR signaling appears to be important in the pathogenesis of cystic biliary dysgenesis. It was shown that biliary epithelial cells were hyper-responsive to the proliferative effect of EGF in a non-orthologous recessive PKD mouse model, the bpk mouse. Moreover, Sato et al. (52) recently demonstrated that, though the biliary epithelia of $p c k$ and control rats displayed similar EGFR localization and signal intensity patterns, an increased proliferative response to EGF was observed in $p c k$ cultured biliary epithelial cells. This effect, in turn, apparently involves the MEK5ERK5 signaling pathway.

It should be noted that abnormal EGFR signaling may be also related to the dysfunctional sodium reabsorption observed in ARPKD. In the distal nephron, Cotton's group (53) demonstrated that EGF signaling through ERK1/2 activation inhibits amiloride-sensitive sodium channels. These investigators reported decreased sodium absorption in $b p k$ mouse-derived cell cultures and suggested that this effect might be medi- ated by increased EGF synthesis and signaling through apical EGFR (54). Potentially conflicting results were obtained in studies using ARPKD cyst-derived epithelial cells. Rohatgi and colleagues (55) showed that these cells not only retained the normal sodium absorptive direction, but also displayed significantly increased sodium reabsorption. These investigators suggested that the increased sodium absorption in ARPKD cells could be partially mediated by the epithelial sodium channel $(\mathrm{ENaC})$ and might contribute to volume expansion and the clinically verified systemic hypertension. Taken together, these results emphasize the importance of carefully considering the studied models before assuming common pathways.

\section{Cyclic AMP in autosomal recessive polycystic kidney disease}

The proliferation of renal cystic epithelial cells has been shown to be stimulated by cyclic AMP (cAMP), an effect that was demonstrated in ADPKD cyst-derived cells (56). In these cells, a cAMP agonist, forskolin, increased the cellular activity of ERK1/2 but had no effect on normal kidney cells. Furthermore, the use of a MEK inhibitor blocked this forskolin-induced proliferation, suggesting that MEK may be the site where cAMP activates the ERK/MAPK cascade (56). A later study showed that in ADPKD cells, but not in normal control cells, cAMP activates ERK through B-Raf (57).

The vasopressin V2 receptor (VPV2R) is believed to be the major cAMP agonist pathway in collecting duct principal cells. Based on this rationale, Torres et al. (58) and Gattone et al. (59) decided to investigate the role of VPV2R antagonists in different PKD animal models. These investigators demonstrated that in the $p c k$ rat, an orthologous model of human ARPKD, the administration of a VPV2R antagonist between 3 and 10 weeks of age reduced renal accumulation of cAMP and inhibited cystic disease development. In addition, 
when performed between 10 and 18 weeks of life, treatment with this antagonist halted disease progression (59). The liver phenotype, however, was not modified. Such observation, in fact, was an expected event, since VPV2Rs are absent in liver. More recently, the protective effect of VPV2R antagonists in $p c k$ rats was shown to be related to inhibition of the Ras/B-Raf/ERK pathway (60).

\section{Conclusion and future prospects}

Although much remains to be elucidated, the understanding of ARPKD molecular and cellular pathogenesis has increased dramatically during the last few years. The identification and characterization of the PKHDI gene, followed by the detailed featuring of the polyductin expression pattern, opened the path for analyses of potentially specific pathogenetic mechanisms. Genetic manipulation of animals, in addition, has now allowed testing potential therapeutic interventions in relevant orthologous models. Such progresses, in turn, are serving and will serve as a knowledge platform to support and guide clinical trials, which might finally offer promising perspectives for ARPKD patients.

\section{References}

1. Guay-Woodford LM, Desmond RA. Autosomal recessive polycystic kidney disease: the clinical experience in North America. Pediatrics 2003; 111: 1072-1080.

2. Capisonda R, Phan V, Traubuci J, Daneman A, Balfe JW, GuayWoodford LM. Autosomal recessive polycystic kidney disease: outcomes from a single-center experience. Pediatr Nephrol 2003; 18 : 119-126.

3. Sharp AM, Messiaen LM, Page G, Antignac C, Gubler MC, Onuchic $\mathrm{LF}$, et al. Comprehensive genomic analysis of PKHD1 mutations in ARPKD cohorts. J Med Genet 2005; 42: 336-349.

4. Bergmann C, Senderek J, Windelen E, Kupper F, Middeldorf I, Schneider $\mathrm{F}$, et al. Clinical consequences of PKHD1 mutations in 164 patients with autosomal-recessive polycystic kidney disease (ARPKD). Kidney Int 2005; 67: 829-848.

5. Osathanondh V, Potter EL. Pathogenesis of polycystic kidneys. Type 1 due to hyperplasia of interstitial portions of collecting tubules. Arch Pathol 1964; 77: 466-473.

6. Desmet VJ. Congenital diseases of intrahepatic bile ducts: variations on the theme "ductal plate malformation". Hepatology 1992; 16: 1069-1083.

7. Zerres K, Mucher G, Bachner L, Deschennes G, Eggermann T, Kaariainen $\mathrm{H}$, et al. Mapping of the gene for autosomal recessive polycystic kidney disease (ARPKD) to chromosome 6p21-cen. Nat Genet 1994; 7: 429-432.

8. Guay-Woodford LM, Muecher G, Hopkins SD, Avner ED, Germino GG, Guillot AP, et al. The severe perinatal form of autosomal recessive polycystic kidney disease maps to chromosome 6p21.1-p12: implications for genetic counseling. Am J Hum Genet 1995; 56: 1101-1107.

9. Onuchic LF, Mrug M, Hou X, Eggermann T, Bergmann C, Zerres K, et al. Refinement of the autosomal recessive polycystic kidney disease (PKHD1) interval and exclusion of an EF hand-containing gene as a PKHD1 candidate gene. Am J Med Genet 2002; 110: 346-352.

10. Onuchic LF, Furu L, Nagasawa Y, Hou X, Eggermann T, Ren Z, et al. PKHD1, the polycystic kidney and hepatic disease 1 gene, encodes a novel large protein containing multiple immunoglobulin-like plexin-transcription-factor domains and parallel beta-helix 1 repeats. Am J Hum Genet 2002; 70: 1305-1317.

11. Ward CJ, Hogan MC, Rossetti S, Walker D, Sneddon T, Wang X, et al. The gene mutated in autosomal recessive polycystic kidney disease encodes a large, receptor-like protein. Nat Genet 2002; 30: 259-269.

12. Hogan MC, Griffin MD, Rossetti S, Torres VE, Ward CJ, Harris PC. PKHDL1, a homolog of the autosomal recessive polycystic kidney disease gene, encodes a receptor with inducible $\mathrm{T}$ lymphocyte expression. Hum Mol Genet 2003; 12: 685-698.

13. Nagasawa $Y$, Matthiesen $S$, Onuchic LF, Hou X, Bergmann $C$, Esquivel E, et al. Identification and characterization of Pkhd1, the mouse orthologue of the human ARPKD gene. J Am Soc Nephrol 2002; 13: 2246-2258.

14. Moser M, Matthiesen S, Kirfel J, Schorle H, Bergmann C, Senderek $\mathrm{J}$, et al. A mouse model for cystic biliary dysgenesis in autosomal recessive polycystic kidney disease (ARPKD). Hepatology 2005; 41: 1113-1121.

15. Bergmann C, Senderek J, Sedlacek B, Pegiazoglou I, Puglia P, Eggermann $T$, et al. Spectrum of mutations in the gene for autosomal recessive polycystic kidney disease (ARPKD/PKHD1). J Am Soc Nephrol 2003; 14: 76-89.

16. Furu L, Onuchic LF, Gharavi A, Hou X, Esquivel EL, Nagasawa Y, et al. Milder presentation of recessive polycystic kidney disease requires presence of amino acid substitution mutations. J Am Soc Nephrol 2003; 14: 2004-2014.

17. Rossetti S, Torra R, Coto E, Consugar M, Kubly V, Malaga S, et al. A complete mutation screen of PKHD1 in autosomal-recessive polycystic kidney disease (ARPKD) pedigrees. Kidney Int 2003; 64: 391403.

18. Menezes LF, Cai Y, Nagasawa $Y$, Silva AM, Watkins ML, Da Silva AM, et al. Polyductin, the PKHD1 gene product, comprises isoforms expressed in plasma membrane, primary cilium, and cytoplasm. Kidney Int 2004; 66: 1345-1355.

19. Ward CJ, Yuan D, Masyuk TV, Wang X, Punyashthiti R, Whelan S, et al. Cellular and subcellular localization of the ARPKD protein; 
fibrocystin is expressed on primary cilia. Hum Mol Genet 2003; 12 : 2703-2710.

20. Wang S, Luo Y, Wilson PD, Witman GB, Zhou J. The autosomal recessive polycystic kidney disease protein is localized to primary cilia, with concentration in the basal body area. J Am Soc Nephrol 2004; 15: 592-602.

21. Zhang MZ, Mai W, Li C, Cho SY, Hao C, Moeckel G, et al. PKHD1 protein encoded by the gene for autosomal recessive polycystic kidney disease associates with basal bodies and primary cilia in renal epithelial cells. Proc Natl Acad Sci U S A 2004; 101: 23112316.

22. Masyuk TV, Huang BQ, Ward CJ, Masyuk Al, Yuan D, Splinter PL, et al. Defects in cholangiocyte fibrocystin expression and ciliary structure in the PCK rat. Gastroenterology 2003; 125: 1303-1310.

23. Praetorius HA, Spring KR. A physiological view of the primary cilium. Annu Rev Physiol 2005; 67: 515-529.

24. Praetorius HA, Spring KR. Bending the MDCK cell primary cilium increases intracellular calcium. J Membr Biol 2001; 184: 71-79.

25. Yoder BK, Hou X, Guay-Woodford LM. The polycystic kidney disease proteins, polycystin-1, polycystin-2, polaris, and cystin, are colocalized in renal cilia. J Am Soc Nephrol 2002; 13: 2508-2516.

26. Nauli SM, Alenghat FJ, Luo Y, Williams E, Vassilev P, Li X, et al. Polycystins 1 and 2 mediate mechanosensation in the primary cilium of kidney cells. Nat Genet 2003; 33: 129-137.

27. Hanaoka K, Qian F, Boletta A, Bhunia AK, Piontek K, Tsiokas L, et al. Co-assembly of polycystin-1 and -2 produces unique cationpermeable currents. Nature 2000; 408: 990-994.

28. Low SH, Vasanth S, Larson CH, Mukherjee S, Sharma N, Kinter MT, et al. Polycystin-1, STAT6, and P100 function in a pathway that transduces ciliary mechanosensation and is activated in polycystic kidney disease. Dev Cell 2006; 10: 57-69.

29. Hou X, Mrug M, Yoder BK, Lefkowitz EJ, Kremmidiotis G, D'Eustachio P, et al. Cystin, a novel cilia-associated protein, is disrupted in the cpk mouse model of polycystic kidney disease. $J$ Clin Invest 2002; 109: 533-540.

30. Woo DD, Tabancay AP Jr, Wang CJ. Microtubule active taxanes inhibit polycystic kidney disease progression in cpk mice. Kidney Int 1997; 51: 1613-1618.

31. Moyer JH, Lee-Tischler MJ, Kwon HY, Schrick JJ, Avner ED, Sweeney WE, et al. Candidate gene associated with a mutation causing recessive polycystic kidney disease in mice. Science 1994; 264: 1329-1333.

32. Taulman PD, Haycraft CJ, Balkovetz DF, Yoder BK. Polaris, a protein involved in left-right axis patterning, localizes to basal bodies and cilia. Mol Biol Cell 2001; 12: 589-599.

33. Sommardahl CS, Woychik RP, Sweeney WE, Avner ED, Wilkinson JE. Efficacy of taxol in the orpk mouse model of polycystic kidney disease. Pediatr Nephrol 1997; 11: 728-733.

34. Brown NE, Murcia NS. Delayed cystogenesis and increased ciliogenesis associated with the re-expression of polaris in $\mathrm{Tg} 737$ mutant mice. Kidney Int 2003; 63: 1220-1229.

35. Siroky BJ, Ferguson WB, Fuson AL, Xie Y, Fintha A, Komlosi P, et al. Loss of primary cilia results in deregulated and unabated apical calcium entry in ARPKD collecting duct cells. Am J Physiol 2006; 290: F1320-F1328.

36. Li JB, Gerdes JM, Haycraft CJ, Fan Y, Teslovich TM, May-Simera H, et al. Comparative genomics identifies a flagellar and basal body proteome that includes the BBS5 human disease gene. Cell 2004; 117: $541-552$

37. Olbrich $\mathrm{H}$, Fliegauf $\mathrm{M}$, Hoefele J, Kispert A, Otto E, Volz A, et al. Mutations in a novel gene, NPHP3, cause adolescent nephronoph- thisis, tapeto-retinal degeneration and hepatic fibrosis. Nat Genet 2003; 34: 455-459.

38. Simons M, Gloy J, Ganner A, Bullerkotte A, Bashkurov M, Kronig C, et al. Inversin, the gene product mutated in nephronophthisis type II, functions as a molecular switch between Wnt signaling pathways. Nat Genet 2005; 37: 537-543.

39. Morgan D, Eley L, Sayer J, Strachan T, Yates LM, Craighead AS, et al. Expression analyses and interaction with the anaphase promoting complex protein Apc2 suggest a role for inversin in primary cilia and involvement in the cell cycle. Hum Mol Genet 2002; 11: 33453350.

40. Lin F, Hiesberger T, Cordes K, Sinclair AM, Goldstein LS, Somlo S, et al. Kidney-specific inactivation of the KIF3A subunit of kinesin-II inhibits renal ciliogenesis and produces polycystic kidney disease. Proc Natl Acad Sci U S A 2003; 100: 5286-5291.

41. Nagano J, Kitamura K, Hujer KM, Ward CJ, Bram RJ, Hopfer U, et al. Fibrocystin interacts with CAML, a protein involved in $\mathrm{Ca}^{2+}$ signaling. Biochem Biophys Res Commun 2005; 338: 880-889.

42. Masyuk TV, Huang BQ, Masyuk AI, Ritman EL, Torres VE, Wang X, et al. Biliary dysgenesis in the PCK rat, an orthologous model of autosomal recessive polycystic kidney disease. Am J Pathol 2004; 165: 1719-1730.

43. Fischer E, Legue E, Doyen A, Nato F, Nicolas JF, Torres V, et al. Defective planar cell polarity in polycystic kidney disease. Nat Genet 2006; 38: 21-23.

44. Mai W, Chen D, Ding T, Kim I, Park S, Cho SY, et al. Inhibition of Pkhd1 impairs tubulomorphogenesis of cultured IMCD cells. Mol Biol Cell 2005; 16: 4398-4409.

45. Gresh L, Fischer E, Reimann A, Tanguy M, Garbay S, Shao X, et al. A transcriptional network in polycystic kidney disease. EMBO $J$ 2004; 23: 1657-1668.

46. Hiesberger T, Bai Y, Shao X, McNally BT, Sinclair AM, Tian X, et al. Mutation of hepatocyte nuclear factor-1beta inhibits Pkhd1 gene expression and produces renal cysts in mice. J Clin Invest 2004; 113: 814-825.

47. Hiesberger T, Shao X, Gourley E, Reimann A, Pontoglio M, Igarashi $P$. Role of the hepatocyte nuclear factor-1beta (HNF-1beta) Cterminal domain in Pkhd1 (ARPKD) gene transcription and renal cystogenesis. J Biol Chem 2005; 280: 10578-10586.

48. Sweeney WE Jr, Avner ED. Functional activity of epidermal growth factor receptors in autosomal recessive polycystic kidney disease. Am J Physiol 1998; 275: F387-F394.

49. Richards WG, Sweeney WE, Yoder BK, Wilkinson JE, Woychik RP, Avner ED. Epidermal growth factor receptor activity mediates renal cyst formation in polycystic kidney disease. J Clin Invest 1998; 101: 935-939.

50. Sweeney WE Jr, Hamahira K, Sweeney J, Garcia-Gatrell M, Frost P, Avner ED. Combination treatment of PKD utilizing dual inhibition of EGF-receptor activity and ligand bioavailability. Kidney Int 2003; 64: 1310-1319.

51. Torres VE, Sweeney WE Jr, Wang X, Qian Q, Harris PC, Frost P, et al. Epidermal growth factor receptor tyrosine kinase inhibition is not protective in PCK rats. Kidney Int 2004; 66: 1766-1773.

52. Sato Y, Harada K, Kizawa K, Sanzen T, Furubo S, Yasoshima M, et al. Activation of the MEK5/ERK5 cascade is responsible for biliary dysgenesis in a rat model of Caroli's disease. Am J Pathol 2005; 166: $49-60$

53. Falin R, Veizis IE, Cotton CU. A role for ERK $1 / 2$ in EGF- and ATPdependent regulation of amiloride-sensitive sodium absorption. Am J Physiol 2005; 288: C1003-C1011.

54. Veizis IE, Cotton CU. Abnormal EGF-dependent regulation of so- 
dium absorption in ARPKD collecting duct cells. Am J Physiol 2005; 288: F474-F482.

55. Rohatgi R, Greenberg A, Burrow CR, Wilson PD, Satlin LM. Na transport in autosomal recessive polycystic kidney disease (ARPKD) cyst lining epithelial cells. J Am Soc Nephrol 2003; 14: 827-836.

56. Yamaguchi T, Pelling JC, Ramaswamy NT, Eppler JW, Wallace DP, Nagao S, et al. cAMP stimulates the in vitro proliferation of renal cyst epithelial cells by activating the extracellular signal-regulated kinase pathway. Kidney Int 2000; 57: 1460-1471.

57. Yamaguchi T, Nagao S, Wallace DP, Belibi FA, Cowley BD, Pelling JC, et al. Cyclic AMP activates B-Raf and ERK in cyst epithelial cells from autosomal-dominant polycystic kidneys. Kidney Int 2003; 63:
1983-1994.

58. Torres VE, Wang X, Qian Q, Somlo S, Harris PC, Gattone VH. Effective treatment of an orthologous model of autosomal dominant polycystic kidney disease. Nat Med 2004; 10: 363-364.

59. Gattone VH, Wang X, Harris PC, Torres VE. Inhibition of renal cystic disease development and progression by a vasopressin $\mathrm{V} 2$ receptor antagonist. Nat Med 2003; 9: 1323-1326.

60. Wang X, Gattone V, Harris PC, Torres VE. Effectiveness of vasopressin V2 receptor antagonists OPC-31260 and OPC-41061 on polycystic kidney disease development in the PCK rat. $J$ Am Soc Nephrol 2005; 16: 846-851. 